(C) 2021 IEEE. Personal use of this material is permitted. Permission from IEEE must be obtained for all other uses, in any current or future media, including reprinting/republishing this material for advertising or promotional purposes, creating new collective works, for resale or redistribution to servers or lists, or reuse of any copyrighted component of this work in other works. 


\section{D Tracking of a River Plume Front with an AUV}

\author{
Diogo Teixeira \\ LSTS, University of Porto \\ Porto, Portugal \\ teixeira.diogodfe.up.pt
}

\author{
Renato Mendes \\ LSTS, University of Porto \\ Collaborative Laboratory +Atlantic \\ Matosinhos, Portugal \\ rpsmelsts.pt
}

\author{
Joana Fonseca \\ KTH Royal Institute of Technology \\ Stockholm, Sweden \\ jfgf@kth.se
}

\author{
João Borges de Sousa \\ Porto, Portugal \\ jtasso@fe.up.pt
}

\begin{abstract}
The problem of the concurrent tracking and mapping of a river plume front with an autonomous underwater vehicle (AUV) is formulated and addressed in the framework of an interdisciplinary approach building on experience in robotics and oceanographic field studies. The problem formulation is targeted at the scientific study of the processes by which the river and the ocean interact. The approach extends previous work in AUV plume tracking to the simultaneous tracking and mapping under different ocean and meteorological conditions. This is done with the help of parameterizable motion control algorithms to enable adaptation to these time-varying conditions. The approach is evaluated in simulation with the help of a highresolution hydrodynamic model. The test plan covers over $\mathbf{3 0 0}$ test cases exercising the most representative combinations of the ocean and meteorological conditions. Lessons learned and future operational deployments are discussed in the conclusions.

Index Terms-AUV River plume, Front-Tracking, Marine Robotics.
\end{abstract}

\section{INTRODUCTION}

A plume is a body of fluid that moves through another one with different properties. The biogeochemical pathways taking place at the interface (front) between these different masses of water have a direct impact, for example, on fisheries, pollution dispersion, and biological production at large. However, we are still far from understanding some of these processes and causal links. This is in part because field studies typically lack the required spatial and temporal resolutions.

Here, we are interested in the study of estuarine river plumes and, in particular, of plume fronts occurring when rivers meet ocean waters. Typically, the study of river plumes is done by using numerical models and satellite imagery (typically, the river plume has a distinct colour or surface temperature), by taking water samples or by doing Conductivity Temperature and Depth (CTD) casts from a manned vessel. These in situ measurements are tedious and lack the required spatial and temporal resolutions. Most remote sensing techniques used to observe river plumes are affected by cloud coverage. Furthermore, daily satellite imagery has poor temporal and spatial

This work was partially funded by the European Union's Horizon 2020 Marine robotics research infrastructure network project under grant agreement No 731103. This support is gratefully acknowledged. resolutions, thus making it difficult to track the evolution of the plume in smaller time scales. Additionally, remote sensing products only provide a $2 \mathrm{D}$ characterization of the plume [1]. AUV based field studies are now addressing some of these limitations because AUVs sample the water column continuously and may adapt the sampling strategy to the observations [2]. Several plume tracking algorithms have been proposed and deployed to accomplish this task. But tracking is just one aspect of the scientific study of river plumes. In fact, the scientific study of plumes is also about finding and mapping them. The problem of finding plumes must be solved before being able to track them. This is relatively easy to solve for river plumes, because of an optical signature that can be detected with the naked eye. The problem of mapping is made simpler if the edge of the plume, the front, is known in realtime because mapping efforts are then limited to a spatial band centered on the front. This observation is the motivation for the developments presented in this paper.

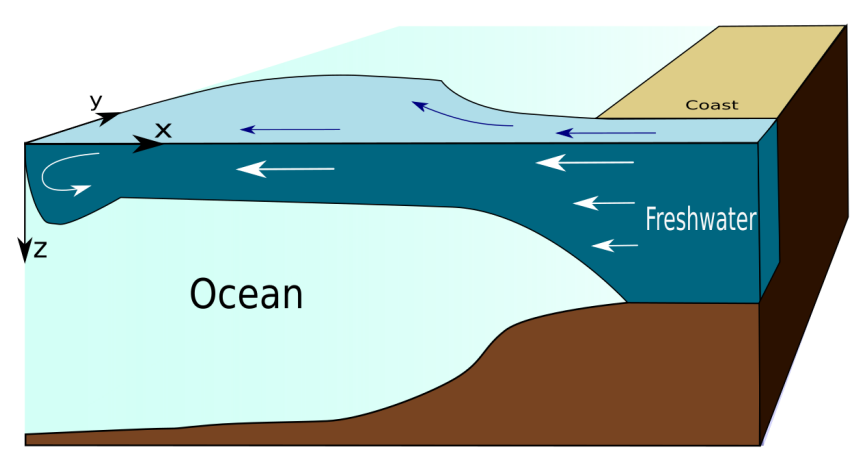

Fig. 1: Diagram depicting a river plume (adapted from [3], Fig. 1).

In this work, we are interested in the processes by which fresh water (lower salinity) from the Douro river (Porto, Portugal) rich in nutrients, sediments and even pollutants interacts with denser ocean water with higher salinity (Figure 1). For this reason, it is important to study and map the dynamics of the plume, which will help predict its influence on the coastal areas surrounding the river mouth. The plume is 
quite dynamic, mainly because of winds and tidal forcing. In practice, the front moves back and forth because of the tides. Surprisingly, or not, the Douro river plume is just a thin layer of fresh water (typical thickness is around 2 meters) moving over ocean waters.

The paper is organized as follows. Section II discusses related work. Section III describes the problem formulation. Section IV is about the overall tracking and mapping approach, with special emphasis on the proposed algorithms and parameterizations of the ocean and meteorological conditions, and how these conditions affect performance. It is also about the deployment of the tracking and mapping algorithms onboard an AUV for field testing. Section V presents the test plan and discusses the results of over 300 simulation tests. Finally, the last section discusses the lessons learned and future work.

\section{RELATED WORK}

The problem of robotic plume front tracking has received some attention over the last decade. Next, we briefly discuss related work.

The problem of tracking of chemical plumes, such as oil spills has been studied, by several authors. A multi-agent system for tracking oil spill plumes was successfully simulated in a realistic test environment by Fahad et al. [4]. This is an estimator-controller system based on the model of a plume with a single-point source that causes propagation of the fluid through advection and diffusion. Li et al. [5] developed a tracking approach that relies on specific properties of the oil plume. Thus, it is not suitable for other marine features such as river plumes. An AUV-mounted sonar, capable of detecting hydrocarbon clouds, is at the core of an oil plume tracking algorithm that detects the plume without crossing it [6] [7]. In our problem, the crossing of the river plume is required and it is not possible to detect it with the use of sonars.

Zhang et al. [8] proposed an approach to detect "a horizontally oriented subsurface hydrocarbon plume" located between depths of 1100 and 1200m caused by the 2010 Deepwater Horizon oil spill in the Gulf of Mexico.

A protocol for adaptive estimation and tracking of harmful algal blooms with a set of unmanned surface vehicles (USVs) using remote sensing data as a prior was developed by Fonseca et al. [9]. The algal bloom is estimated as a circumference that the robots will circumnavigate. A circumnavigation control law is applied to all robots with the goal of converging to the boundary and maintaining an equal distribution across the circumference. The algorithm can be modified to take into consideration ellipsoid shapes. In our work, we do not consider assumptions on the shape of the plume, other than being continuous.

Ocean isolines of salinity or temperature have been successfully tracked by marine robots. A segment of the open-ocean Northern Pacific Subtropical Front (STF) was autonomously tracked with an AUV running a novel "front core tracking" algorithm [10]. The parameters of the salinity signature of the front were first estimated to inform the tracking algorithm. The proposed algorithm uses a novel model for the salinity signature of the front. Previous algorithms model the salinity signature with a linear function connecting the boundaries of the front. Outside these boundaries salinity is considered to be constant. Other algorithms model this signature as a smooth function, with the plume front being located at the peak of the salinity gradient. Belkin et al. [10] use the front edges instead of the front axis to guide the "front core tracking" algorithm. The vehicle follows the front axis by remaining inside the front. This strategy has two main advantages: the distance travelled by the vehicle is minimized because the front is not fully crossed like in a typical zigzag approach and progression along the front progression is faster. Furthermore, the risk of "losing the front" is small because the AUV is kept inside the front. Here, we are interested in mapping the plume of the Douro river. This cannot be accomplished by keeping the AUV inside the front. Furthermore, the Douro plume has a small front width. This precludes the use of the "front core tracking" algorithm

An upwelling front-tracking algorithm was field-tested with an AUV in Monterey Bay, CA [11]. The algorithm uses a yoyo vertical trajectory to measure the temperature at different depths while crossing the front at different locations. The multiple front detection points are used to estimate the progression of the front. A similar yoyo trajectory will be used in our implementation to measure salinity at different depths. Analogous to the described system, multiple crossings of the front are then used the estimate the progression of the front.

A front-tracking algorithm for a thermal plume caused by a nuclear power plant outflow was implemented on a USV and successfully deployed in the field [12]. The algorithm proposed an adaptive zigzag trajectory based on measurements of six different variables. These measurements are then used by a plume indication function that signals if the vehicle is inside or outside of the plume. The authors conclude that the dependence of this function on six different variables increases the robustness of the tracking system. A similar zigzag like trajectory is used in our approach.

Two approaches for static plume tracking and mapping were studied by Cannell and Stilwell [13]. In the first, an AUV travels a fixed path in the study area and then returns a probabilistic distribution of the plume based on the measurements and path of the agent. The second approach is a non-parametric boundary tracking algorithm that first runs a classification phase that identifies the two different regions and proposes a probability distribution of the plume's boundary that is then used to guide the agent along the front. These approaches are not applicable to the dynamic plume of the Douro river.

Zigzag like trajectories for tracking marine fronts were experimentally validated with ASVs in [14]. The tracking system generates an initial path for the vehicle based on a previous characterization of the front and using observations from different remote sensing agents.

An algorithm for tracking the Douro river plume with multiple underwater and air vehicles was successfully implemented and field tested in the field with success is presented in [1]. Air vehicles are used to find the plume and underwater 
vehicles are used to cooperatively track different segments of the plume using a combination of zigzag and yoyo trajectories. Expanding on this implementation, our approach estimates the front direction and adapts the vehicle's trajectory to retrieve the maximum number of perpendicular transects to the front with the goal of improving the mapping performance. The novel aspects of the approach include an exploration phase, in which essential parameters of the plume are estimated, the adaptation strategy, encompassing concurrent tracking and mapping, and the use of several parameters to enable the user to select and configure the new algorithms, as well as to pick the initial deployment locations, according to the environmental conditions.

\section{Problem Statement}

Here, we formulate the problem addressed in this paper. We are interested in the study of the Douro river plume, more specifically in the salinity and associated temperature maps. Thus, we need a few definitions.

The river plume is defined as a scalar field, Plume, evolving with time:

$$
\text { Plume }: D \subset \mathbb{R}^{4} \rightarrow \mathbb{R}^{2}
$$

where $D$ is a closed set. Plume has two variables of interest, salinity, $S$, and temperature, $T$ :

$$
\text { Plume }(x, y, z, t)=[T(x, y, z, t), S(x, y, z, t)]
$$

No assumptions on the type of mathematical model that describes these variables are made. The evolution of Plume is determined by a set of parameters Parameters Plume $^{=}$ $\left\{\rho_{1}, \ldots, \rho_{n}\right\}$. The set of parameters describes wind, tide, salinity profile of the front, velocity of the plume, among others, that directly influence the shape and position of the plume. Some of these parameters are unknown, others are known a priori, while others are calculated during the mission.

We do not have direct access to Plume function, only to point-wise measurements taken by an AUV along a trajectory $\operatorname{Traj}:\left[t_{0}, t_{f}\right] \rightarrow \mathbb{R}^{3}$.

The position, $p$ of the AUV at time $t$, is $p(t)=$ $[x(t), y(t), z(t)]$. The evolution of $p$ is governed by the equation of motion:

$$
\dot{p}(t)=f(p(t), u(t), v(t), t), \quad u(t) \in U, \quad v(t) \in V
$$

where $u(t)$ and $v(t)$ are respectively the AUV control inputs and the velocity of the currents; $U$ and $V$ are compact sets.

The measurements of salinity and temperature taken by the AUV at time $t$ are, respectively, $\hat{S}(p(t), t)$ and $\hat{T}(p(t), t)$.

The streams of salinity and temperature measurements, taken by the AUV up to time $t$ are respectively

$$
\begin{aligned}
& \text { StreamS }(t):\left[t_{0}, t_{f}\right] \rightarrow C, \operatorname{Stream} S(t)=\hat{S}(\operatorname{Traj}(t), t) \\
& \operatorname{Stream}(t):\left[t_{0}, t_{f}\right] \rightarrow C, \operatorname{StreamT}(t)=\hat{T}(\operatorname{Traj}(t), t)
\end{aligned}
$$

where $C$ is the space of continuous functions $[\mathbb{R} \rightarrow \mathbb{R}]$.

The problem addressed in this paper is:

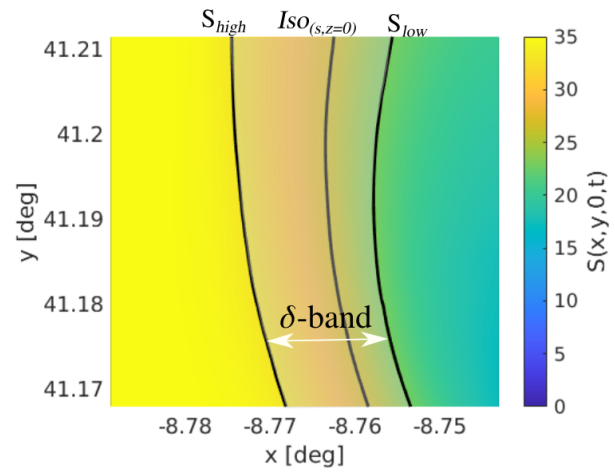

Fig. 2: Representation of the $\delta$-band defined by the salinity values $S_{\text {low }}$ and $S_{\text {high }}$.

Problem 1. Given an initial AUV position $p\left(t_{0}\right)$ and a set of parameters, Parameter Plume $_{\text {: }}$

i) Track a $\delta$-band centered on the $s$ isoline of the plume located at depth $z_{d}, I s o_{\left(s, z_{d}=0\right)}=\{(x, y, z, t)$ : $\left.S\left(x, y, z_{d}, t\right)=s\right\}$.

ii) Map the plume in this $\delta$-band defined by two salinity threshold values, $\left[S_{\text {low }}, S_{\text {high }}\right]$ (Figure 2).

iii) Ensure that the direction of travel of the AUV when crossing the front is perpendicular to $I s o_{\left(s, z_{d}\right)}$ for half of the total crossings.

iv) Map the isosurface where $S(x, y, z, t)=s$ in the $\delta$ band

The requirement for perpendicular crossings of the front comes from the observation that non-perpendicular crossings can lead to significant distortions in the calculation of the plume characteristics such as spreading width, length of hydrodynamic mixing zone and minimum dilution.

\section{APPROACH}

The approach for tracking and mapping the Douro river plume is based on three different motion adaptation algorithms and on a front estimation procedure. The algorithms are tuned with a few parameters to enable user-selected adaptation to ocean and meteorological conditions.

\section{A. Algorithms}

We propose three algorithms to track and map the Douro plume front (Figure 3 ). These algorithms are basically characterized by the shape of the resulting paths. The paths depicted in this figure are horizontal projections of 3D yoyo paths to be performed by the vehicle.

The Zigzag is characterized by two straight line paths that make an angle $\alpha$ when the paths cross inside the plume. In addition, this algorithm also guarantees, up to some error, that the front is crossed in a direction perpendicular to it when the vehicle enters the plume.

The Alternative Zigzag adds a straight line segment of length $D_{\text {out }}$ parallel to the front for excursions outside the plume. This trajectory results in a faster front progression since 


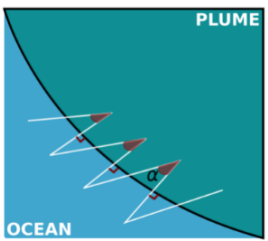

(a) Zigzag.

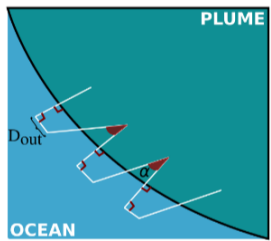

(b) Alt. Zigzag.

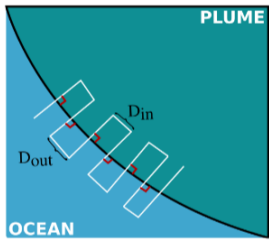

(c) Lawn Mower.
Fig. 3: Schematic of the algorithms for tracking and mapping the Douro river plume.

it increases the space between front crossing points compared to the Zigzag trajectory.

The ideal crossing of the plume should be perpendicular to the front, as this is the situation that yields the ideal conditions for sampling the salinity profile of the plume. For this reason, a path that always traverses the front with perpendicular crossings would be ideal. This is achieved by also performing a parallel transect to the front of length $D_{i n}$ when moving inside the plume. This path is referred to as Lawn Mower. Change of directions are triggered when the salinity thresholds $\left[S_{\text {high }}, S_{\text {low }}\right]$ are met and a minimal distance $D_{\min }$ or a maximum distance $D_{\max }$ from the front are reached.

\section{B. Front estimation and detection}

Initially, the vehicle does not have any information on the shape and parameters of the front. This poses two different problems. Firstly, an initial trajectory must be capable of crossing the front without any previous information on its shape. Secondly, the shape of the front must be predicted in order to determine the perpendicular segments to it.

The problem of finding the front is addressed by having the vehicle performing a classical zigzag trajectory with an angle $\alpha$ between all segments of the trajectory. The first three front crossings enable the estimation of some essential parameters of the front. After these three crossing points, the trajectory adaptation algorithms are initiated.

For the front's prediction, the last and third last front crossing points, $P_{\text {cross }_{n}}$ and $P_{\text {cross }_{n-2}} \in \mathbb{R}^{2}$, are used to perform a linear prediction. The vector, $\vec{f}=\left[f_{1}, f_{2}\right]^{T}$, is formed by the two points and represents the estimation of the front's direction, $\gamma$. Thus,

$$
\begin{aligned}
& \vec{f}=\vec{P}_{\text {cross }_{n}}-\vec{P}_{\text {cross }_{n-2}}, \\
& \gamma=\angle \vec{f}=\operatorname{atan} 2\left(f_{2}, f_{1}\right) .
\end{aligned}
$$

The direction $\gamma$ is used to compute the perpendicular segments. The error between the true perpendicular direction and the predicted is $\epsilon$.

Figure 4 shows a schematic example of the first zigzag trajectory and the process of predicting the direction of the front after detecting three crossing points.

Observe that the plume is a thin layer of fresh water located at the surface. Hence, the first crossing of the plume is done at the surface and will determine the reference salinity value

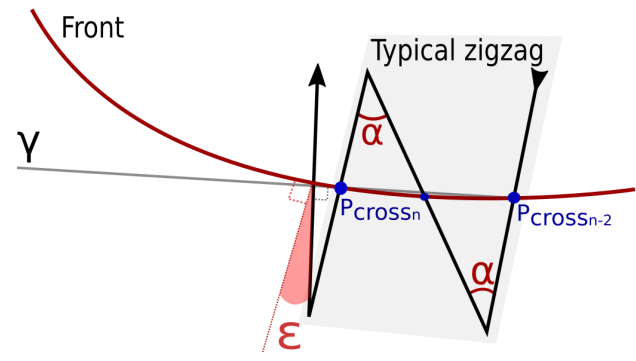

Fig. 4: Schematic representation of the typical zigzag trajectory and the first front estimation $\gamma$ after three crossings of the front.

$s$ for the front. This reference salinity value is calculated for every new mission.

Since the front is characterized by a steep change of the salinity field, the first survey at the surface is used to find the maximum derivative of the sampled values and the associated instant $t_{\max }$ where that maximum rate of change occurs. Let $s$ be the value of salinity at instant $t_{\max }$ (7). This provides an estimate of the maximum gradient of the salinity field on that region.

$$
\begin{aligned}
t_{\max } & =\left\{t: \max \left(\frac{\partial \hat{S}(x, y, 0, t)}{\partial t}\right)\right\} \\
s & =\hat{S}\left(x, y, z, t_{\max }\right)
\end{aligned}
$$

\section{C. $3 D$ mapping}

The mapping of the plume has to be done in 3 dimensions. This is accomplished by having the vehicle performing a yoyo trajectory, defined by the pitch angle $\theta=15^{\circ}$ between the path and the horizontal plane and cycling between the surface and some maximum depth $z_{\max }$. The nominal value for $z_{\max }$ is chosen to $10 \mathrm{~m}$ because the thickness of the plume is in the order of $2 \mathrm{~m}$ in nominal conditions. The plume crossing logic is enabled only when the depth of the vehicle exceeds 25 centimeters $(z>0.25 \mathrm{~m})$.

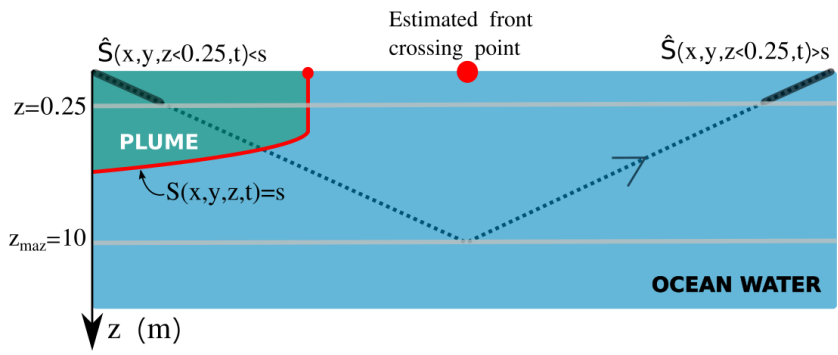

Fig. 5: Vertical profile of the AUV yoyo trajectory (black dashed line) while crossing the front. The estimated crossing point is the middle point between the surface locations.

Figure 5 shows a typical situation where the vehicle crosses the front when starting inside the plume. A plume crossing, $P_{\text {cross }}$, is declared as the middle point between the two last surface locations, $P_{\text {surface }} \in \mathbb{R}^{2}$ (8). 


$$
P_{\text {rross }_{n}}=\frac{P_{\text {surface }_{i}}+P_{\text {surface }_{i-1}}}{2}
$$

\section{Environmental and algorithm parameters}

In this section, we discuss the parameters affecting the performance of the algorithms.

We consider three environmental parameters: tide, wind and river outflow. Tides determine the time window during which the mission should take place -6 hours during the ebb phase. In what follows we consider that missions take place during this phase.

Wind plays an important role in determining the shape of the plume. There are three main shapes of the plume are caused by Southerly, Northerly, and East or light wind conditions (Figure 8). This knowledge is used to select the initial position of the AUV for any sampling mission. The initial direction of the vehicle is parallel to the coast. If there is a northerly wind the vehicle starts in a northward direction. Conversely, if a southerly wind is identified, we start the vehicle in a southward direction. The initial location is always fairly close to the river mouth to guarantee that the vehicle is inside the plume. Lastly, the southwest wind causes a circular-shaped plume where the vehicle can start in both directions because it is always expected to cross the plume.

The levels of the river outflow are a major consideration when it comes to field deployments of the vehicle. It may happen that the plume is not well defined for some regimes. This typically happens when the river discharge is either too small or too large.

Next, we discuss the trajectory and mapping parameters used to tune the algorithms. These will directly influence the behavior and performance of the system. The settings of these parameters are defined by the user before deploying the AUV. The results presented in section $\mathrm{V}$ provide insights into optimal parameter selection. The trajectory parameters are presented in Table I.

\begin{tabular}{|c|c|c|}
\hline Algorithm & \multicolumn{2}{|c|}{ Parameters } \\
\hline Zigzag & \multicolumn{2}{|c|}{$\alpha$} \\
\hline Alt. Zigzag & $D_{\text {out }}$ & $\alpha$ \\
\hline Lawn Mower & $D_{\text {out }}$ & $D_{\text {in }}$ \\
\hline
\end{tabular}

TABLE I: Trajectory parameters.

The mapping parameters are presented next. These determine the mapping behavior of the vehicle. These are: the maximum vertical distance $\left(z_{\max }\right)$; the yoyo/pitch angle $(\theta)$; plume thresholds $\left(S_{\text {high }}, S_{\text {low }}\right)$; and the minimum and maximum distance covered by the vehicle after crossing the front $\left(D_{\min }, D_{\max }\right)$. Table II presents the parameters used for the case of the Douro river plume.

\begin{tabular}{|c|c|}
\hline Parameter & Value \\
\hline$z_{\max }$ & $10 \mathrm{~m}$ \\
\hline$\theta$ & $15^{\circ}$ \\
\hline$\left[S_{\text {high }}, S_{\text {low }}\right]$ & {$[33,23]$} \\
\hline$\left[D_{\min }, D_{\max }\right]$ & {$[500,750] \mathrm{m}$} \\
\hline
\end{tabular}

TABLE II: Parameters used for mapping the Douro river plume.

\section{E. Implementation - LSTS toolchain}

The algorithms presented in this section were deployed on a Light Autonomous Underwater Vehicle LAUV with the help of the open-source LSTS software toolchain developed by the Laboratório de Sistemas e Tecnologia Subaquática [15].

The toolchain includes DUNE and Neptus, which communicate with the IMC message protocol [16], as depicted in Figure 6.

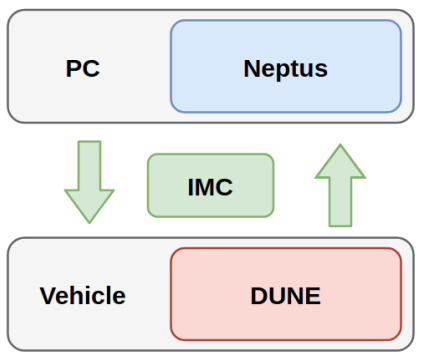

Fig. 6: LSTS toolchain: components and interactions.

Neptus is graphical user-interface supporting planning and execution control [17]. The LAUV communicates with Neptus via acoustic modems (when underwater) or via Wi-Fi (when at the surface).

DUNE is the LAUV onboard software handling navigation, control, communications, logging and interactions with the vehicle's hardware. DUNE provides tasks able to interact with all sensors and actuators of the LAUV as well as the software infrastructure to deploy additional control and navigation tasks.

A new control task was developed to deploy the fronttracking algorithms as well as the front estimation calculations. This task communicates with other tasks by using four IMC messages, as described in Figure 7.

The low-level control of the vehicle is done with the help of two other control tasks, also called maneuver controllers: YoYo and GoTo. The new control task sends one message to each maneuver controller. The GoTo maneuver will control the vehicle to move to a given waypoint and YoYo maneuver is about implementing a yoyo trajectory. The new control task consumes messages encoding the estimated state and salinity measurements, that are periodically broadcast by the two other tasks depicted in Figure 7.

DUNE has a simulation mode to facilitate the integration and validation of new onboard software with the help of models of the sensors and actuators. This is why the two tasks sending messages to the new control task can get data from simulation models and/for recorded files or from the hardware, in field deployments. This mode was used to validate the algorithms developed in this work prior to field deployments.

\section{Results}

We developed a simulation environment developed in MATLAB to test and validate the proposed approach. We used the DELFT3D ocean model [18] to generate salinity and water flow data used in these simulations. 


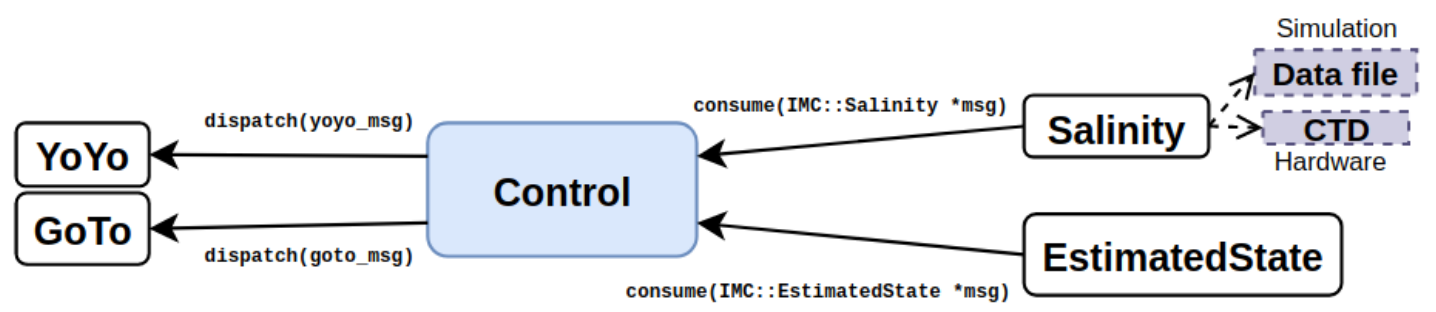

Fig. 7: Control interactions - IMC messages exchanged with other DUNE tasks.

\section{A. Test plan}

The algorithms were tested in three main shapes of the plume determined by different wind conditions: Southerly; Northerly; and, Light Wind. Typically, Southerly winds result in faster propagation velocities for the plume.
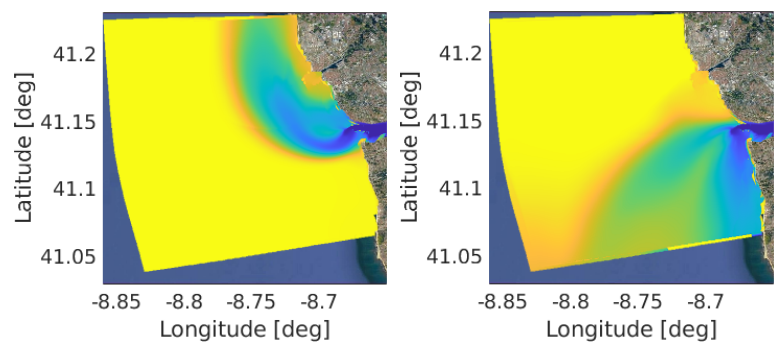

(a) $9^{\text {th }}$ May $2016-S$ plume.

(b) $15^{\text {th }}$ May $2016-N$ plume.

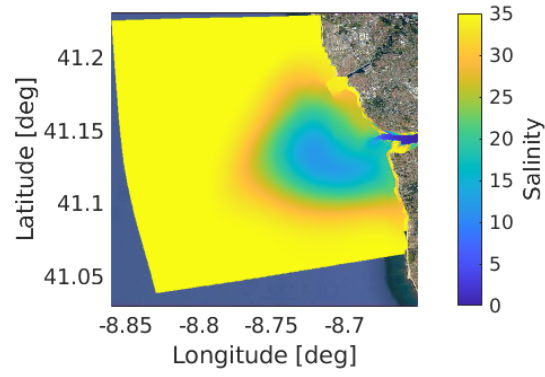

(c) $2^{\text {nd }}$ May 2017 - LW plume.

Fig. 8: Typical plume patterns for Southerly-S (a), Northerly-N (b), and East and/or light wind-LW (c) conditions.

Table III describes 307 test cases grouped into 7 different test sets, $\mathbf{A}$ to $\mathbf{D}$, covering different plume conditions and parameters. Plume conditions included: i) static or dynamic plumes; ii) 2D or 3D tracking; and, iii) the presence (or not) of ocean currents. Static plumes do not represent a realistic test case. Nevertheless, these tests proved very useful to study the properties of the three algorithms.

Test sets $\mathbf{A}$ and B.1 included nine tests each exercising the three algorithms for the three different plume patterns. B.2 exercises the effect of the AUV velocity $v$ (ranging between 0.5 to $5 \mathrm{~m} / \mathrm{s}$ ). C.2 exercises the effect of the length of the yoyo cycle (distances between consecutive samples at the surface) ranging from $3.7 \mathrm{~m}$ to $458.1 \mathrm{~m}$. D tests different values of the trajectory parameters $\left(\alpha \in[20,70]^{\circ}, D_{\text {out }} \in\right.$ $[100,800] \mathrm{m}$ and $\left.D_{i n} \in[100,800] \mathrm{m}\right)$. The large number of
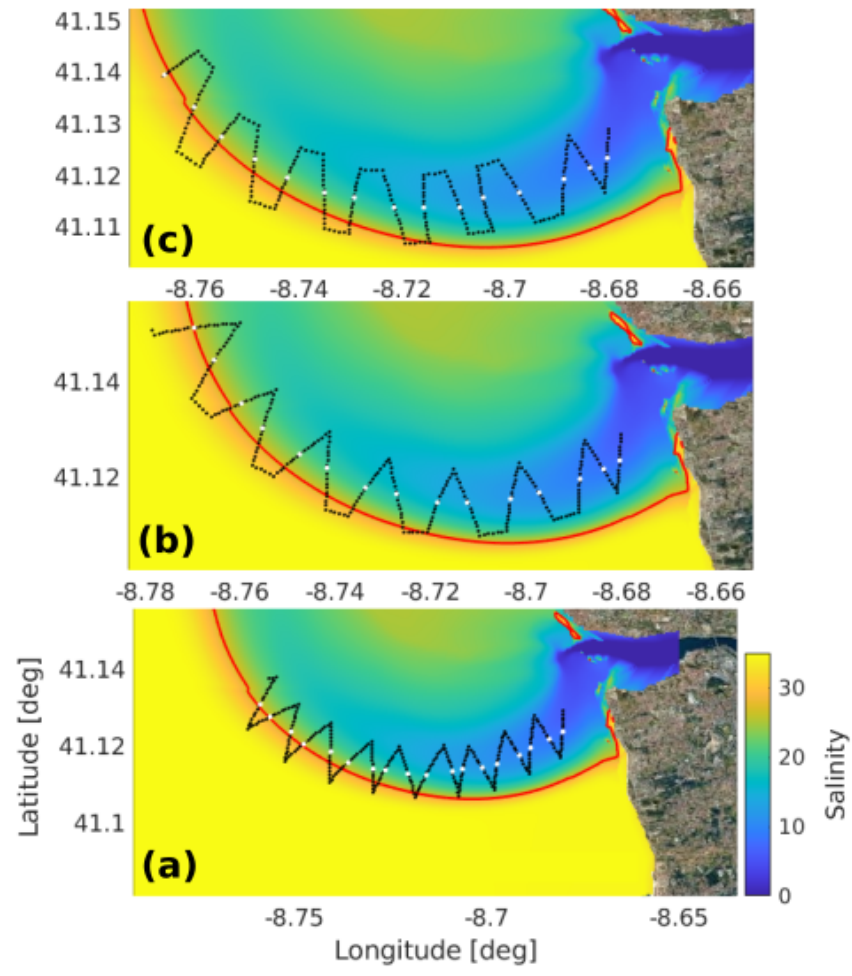

Fig. 9: Results from set C.1 - 6-hour mission from 4:30 to 10:30 on the S plume. Algorithms: (a) Zigzag, (b) Alt. Zigzag, (c) Lawn Mower. The vehicle position at surface level $(z<$ $0.25)$ is represented in black. The orange line represents the plume's front.

tests for test set $\mathbf{D}$ targeted the development of guidelines for a decision support system to help the user in picking these parameters to best suit the testing conditions.

Figure 9 shows simulation runs for test set C.1 with a $S$ plume. As we can see, the AUV is successfully tracking the plume with the three algorithms. Also, note that this is the fastest propagating plume and that the algorithms are still capable of tracking the front.

Next, we present the performance metrics used in these simulations before briefly discussing the results for the seven test sets.

\section{B. Performance metrics}

We consider three performance metrics: 


\begin{tabular}{|c|c|c|c|c|c|c|c|}
\hline & \multicolumn{4}{|c|}{ Plume } & & & \\
\hline Set & Static & Dynamic & 2D & 3D & $\begin{array}{c}\text { Ocean } \\
\text { Currents }\end{array}$ & $\begin{array}{l}\text { \# of } \\
\text { tests }\end{array}$ & Description \\
\hline $\mathbf{A}$ & $\checkmark$ & & $\checkmark$ & & & 9 & Test performance for a static plume \\
\hline B.1 & & $\checkmark$ & $\checkmark$ & & & 9 & Test performance for a dynamic plume \\
\hline B.2 & & $\checkmark$ & $\checkmark$ & & & 16 & Test the effects of the AUV velocity $v$ \\
\hline C.1 & & $\checkmark$ & & $\checkmark$ & & 9 & Test performance for 3D tracking of a dynamic plume \\
\hline C.2 & $\checkmark$ & & & $\checkmark$ & & 30 & Test different yoyo configurations \\
\hline $\mathbf{D}$ & $\checkmark$ & $\checkmark$ & $\checkmark$ & $\checkmark$ & & 216 & Test trajectory parameters $\left(\alpha, D_{\text {out }}\right.$ and $\left.D_{\text {in }}\right)$ \\
\hline $\mathbf{E}$ & & $\checkmark$ & & $\checkmark$ & $\checkmark$ & 18 & Test the effects of ocean currents \\
\hline
\end{tabular}

TABLE III: Test plan.

1) The quality of each mission is measured by the average error of the front prediction $\epsilon_{\text {avg }}$ presented below where $i$ refers to the error in each transect. We also label each individual transect as optimal if $\left\|\epsilon_{i}\right\|<20^{\circ}$.

$$
\epsilon_{\text {avg }}=\frac{1}{n} \sum_{i=1}^{n}\left\|\epsilon_{i}\right\|,
$$

2) The speed of progression along the front $v_{f p}(\mathrm{~m} / \mathrm{s})$ is given by the length of the front mapped so far (calculated from successive front crossings) divided by the duration of the mission $t_{M}$ :

$$
v_{f p}=\frac{1}{t_{M}} \sum_{i=2}^{n}\left\|\vec{P}_{\text {rross }_{i}}-\vec{P}_{\text {cross }_{i-1}}\right\| .
$$

3) The percentage of optimal (perpendicular) crossings for a given mission.

\section{Test sets A, B.1 and C.1: Performance of the algorithms}

Test sets A, B.1 and C.1 targeted a comparative study of the algorithms under the same plume patterns and tuning parameters. The performance ranking of the algorithms under these conditions is briefly summarized in Table IV.

\begin{tabular}{|c|c|c|c|}
\cline { 2 - 4 } \multicolumn{1}{c|}{} & Zigzag & Alt. Zigzag & Lawn Mower \\
\hline Estimated average error & 1 & 2 & 1 \\
\hline \# of optimal crossings & 2 & 3 & 1 \\
\hline Front progression & 2 & 1 & 2 \\
\hline
\end{tabular}

TABLE IV: Rankings of the algorithms: worst (3) to best (1).

\section{Test set B.2: Effects of the AUV velocity}

Test set B.2 aimed at studying how the vehicle velocity impacts tracking performance. This was done for the fast $S$ and slow $N$ propagating fronts. The results are shown in Figure 10.

Observe that some AUV velocities used in these tests are not feasible. However, these results provide insights into the role of the vehicle's speed in the tracking mission. Starting with the fast front (S plume) represented in blue, we observe that the increase in velocity produces a better front prediction that converges to the performance on the static plume (dashed line). As expected, the increase in the AUV velocity correlates to an increase in performance for fast moving plumes; this effect is almost negligible for slow moving plumes.

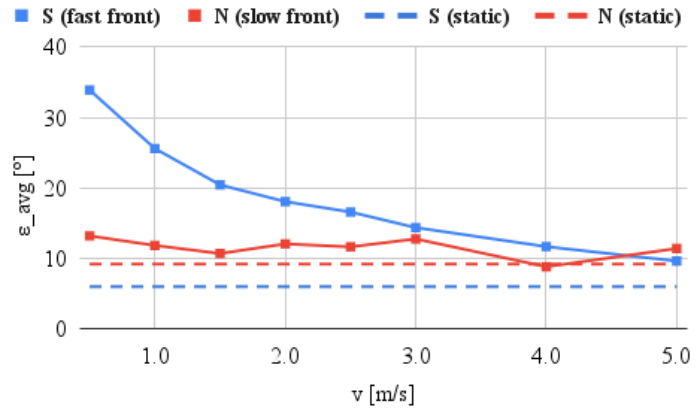

Fig. 10: Average error, $\epsilon_{\text {avg }}$, for different vehicle velocities, $v$.

\section{E. Test set C.2: Effects of the length of yoyo trajectory cycle}

Test set C.2 aims at studying the effects of the length of the yoyo cycle on performance. In fact, the vehicle samples the plume's surface one time per yoyo cycle. For example, the distance between consecutive samples at the surface is $d_{\text {surface }}=74.6 \mathrm{~m}$ for a pitch angle of $\theta=15^{\circ}$ and with $z_{\max }=10 \mathrm{~m}$

As expected, a general upward tendency in $\epsilon_{\text {avg }}$ is verified with the increase of $d_{\text {surface }}$ (Figure 11). However, it is important to note that this trend is not noticeable when $d_{\text {surface }}<100 \mathrm{~m}$. This provides good evidence that in this range, the 3D mapping of the plume does not heavily influence the tracking performance.

One would expect that the vehicle would not be able to track the plume for larger values of $d_{\text {surface }}$ (e.g., $400 \mathrm{~m}$ ). Surprisingly, this was not the case for tests with a static plume.

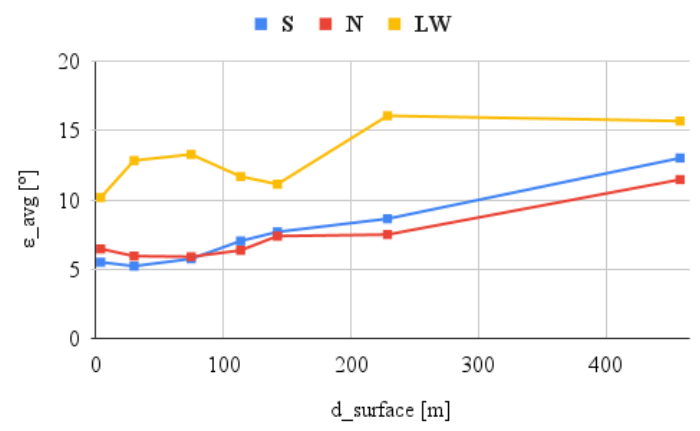

Fig. 11: Effect of the distance between surface points $d_{\text {surface }}$ on the average error $\epsilon_{\text {avg }}$ for a Zigzag trajectory. 


\section{F. Test set D: Effects of different trajectory parameters}

Test set $\mathbf{D}$ evaluated the effects of variations of the $\alpha$ trajectory parameter for static $2 \mathrm{D}$ and dynamic $3 \mathrm{D}$ plumes.

In the static 2D tests, larger $\alpha$ angles (and larger $D_{i n}$ and $D_{\text {out }}$ distances) result in larger distances between crossing points, thus reducing tracking performance (measured by average error variation on $\alpha$ ) as shown in Figure 12a for the Zigzag algorithm. Similar qualitative results were obtained for the other parameters and strategies.

In the dynamic 3D tests, the close-to-linear relations obtained for the static 2D tests are no longer valid. The best performance is not achieved when $\alpha=20^{\circ}$ but when $\alpha \in[30,50]^{\circ}$ (Figure 12b). Thus, the best performance is not guaranteed when the front crossing points are at a minimal distance from each other. In fact, the locations of $P_{\text {cross }}$ are not as accurate as before and the prediction with closer points does not always result in a more accurate description of the front's orientation.

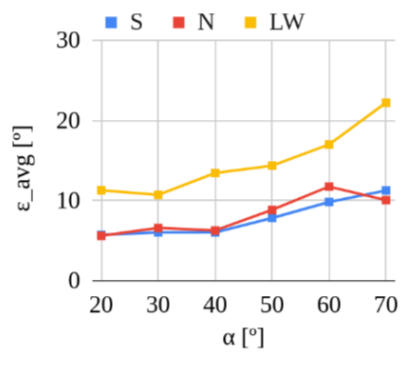

(a) Static 2D plume.

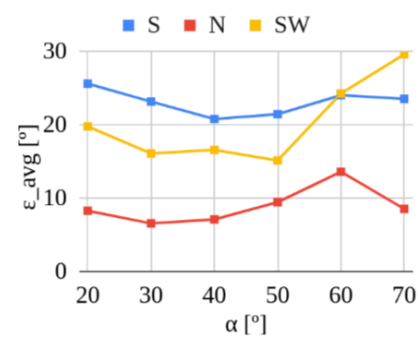

(b) Dynamic 3D plume.
Fig. 12: Effect of the trajectory angle, $\alpha$, on the average error, $\epsilon_{\text {avg }}$ for a Zigzag strategy.

The effects on the speed of progression $v_{f p}$ are as expected for all test cases. The speed of progression increases with larger distances between crossing points which, in turn, increases with $\alpha$ (Figure 13).

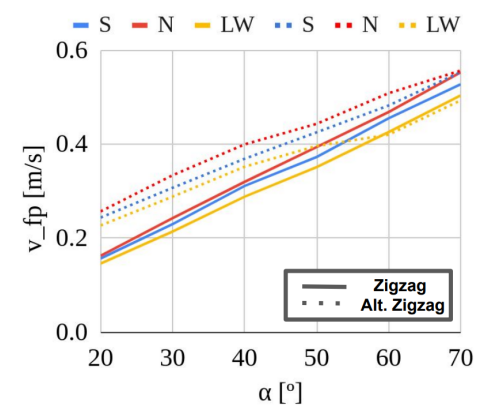

Fig. 13: Effect of the trajectory angle $\alpha$ on the speed of progression $v_{f p}$.

\section{G. Test set $\boldsymbol{E}$ : effects of ocean currents}

The motions of the AUV are affected by ocean currents (see Equation 3). Moreover, the AUV used in this work does not have sensors to measure the velocity with respect to the seabed. This affects the trajectory tracking performance. To minimize this effect, we used the path correction method (provided by DUNE) when the AUV reaches the surface: i) the position of the AUV is reset with the GPS coordinates when the AUV breaches the surface; ii) the AUV is commanded to move to the real surfacing position before diving again.

Test set $\mathbf{E}$ includes runs without and with the path correction method. Results are compared to the ones from test set C.1. Table V shows the effect of the ocean currents on the estimated average error.

As expected, ocean currents have an impact on the tracking performance. Overall, the path correction method mitigates these effects. However, it does not completely correct the trajectory of the vehicle. In some cases it does not even improve performance.

Moreover, these tests show that plume tracking is not compromised by the effects of the ocean currents. Figure 14 shows one run with the same parameters and plume conditions used in the run from Figure 9, but taking into account the effects of the water velocity. This simulation is the most realistic of the overall test plan.

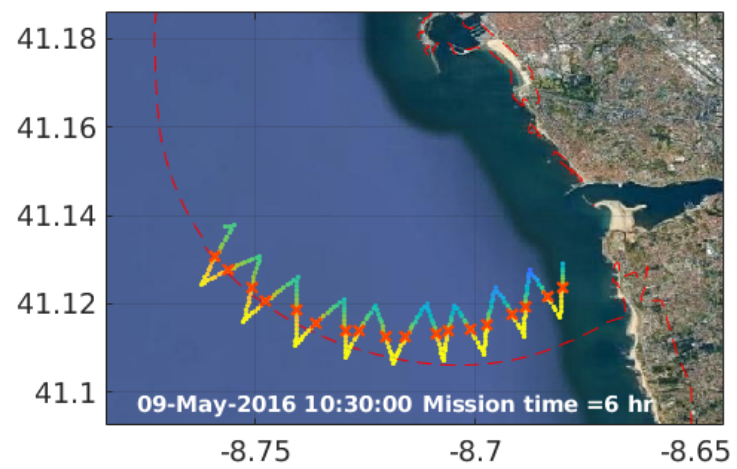

Fig. 14: Simulation run from simulation set E. Zigzag algorithm in the presence of ocean currents (satellite image of the Douro estuary as background).

\section{H. Salinity maps}

One of the main goals of the AUV mission is to map the plume. The maps built from the sampled salinity data provide a good characterization of the plume in the band of interest. Figure 15 shows the salinity map built with the data collected during the mission represented in Figure 14. Transitions from the plume to ocean waters are easily identified in this map.

The mapping results for all the tests are very similar to ones obtained in previous field tests. The validation of these results should be done with extensive field testing scheduled for September 2021.

\section{CONCLUSIONS}

The problem of tracking and mapping a river plume front with an AUV is formulated and solved with the help of novel trajectory adaptation algorithms. The overall approach was evaluated and tested in simulation using a high-resolution hydrodynamic model of the plume. The test plan included 


\begin{tabular}{|c|c|c|c|c|c|c|c|c|c|}
\cline { 2 - 10 } \multicolumn{1}{c|}{} & \multicolumn{3}{c|}{ Zigzag } & \multicolumn{3}{c|}{ Alt. Zigzag } & \multicolumn{3}{c|}{ Lawn Mower } \\
\hline Plume & OC [\%] & PC [\%] & $\Delta[\%]$ & OC [\%] & PC [\%] & $\Delta[\%]$ & OC [\%] & PC [\%] & $\Delta[\%]$ \\
\hline N & 60 & 69 & $\mathbf{9}$ & 101 & 70 & $\mathbf{- 3 1}$ & 8 & -7 & $\mathbf{- 1 5}$ \\
\hline S & 37 & 28 & $\mathbf{- 9}$ & 40 & 35 & $\mathbf{- 5}$ & -3 & -9 & $\mathbf{- 6}$ \\
\hline LW & 37 & -5 & $\mathbf{- 4 2}$ & 3 & -39 & $\mathbf{- 4 2}$ & -12 & -36 & $\mathbf{- 2 4}$ \\
\hline
\end{tabular}

TABLE V: Variation of the average estimation error, $\epsilon_{\text {avg }}$, compared to the test where ocean currents are zero. $O C$ - Only ocean currents are taken into account; $P C$ - Path correction method active; $\Delta$ - Difference between the two results.

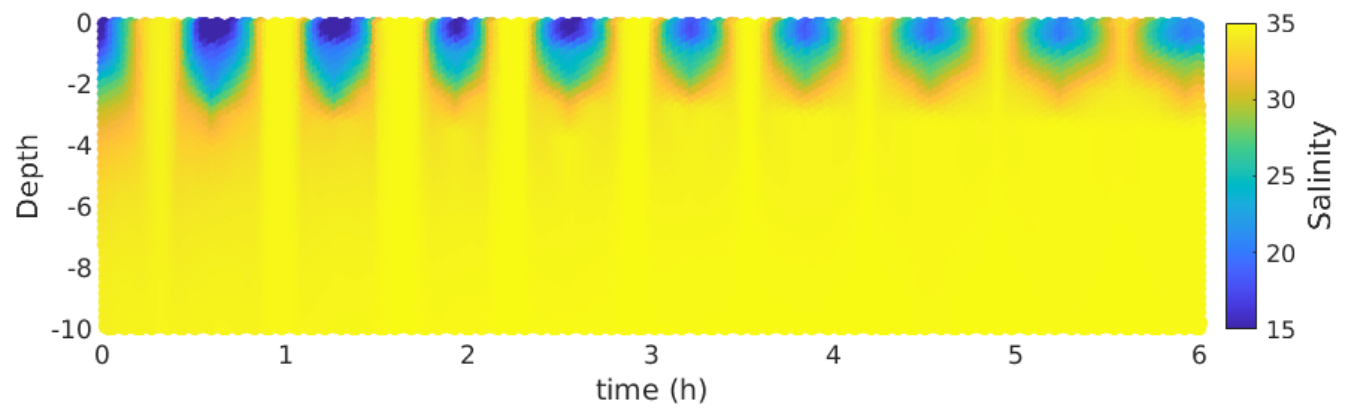

Fig. 15: Salinity map obtained from the data collected in the test represented in Figure 14.

307 test cases covering the most representative environmental conditions. This was an interdisciplinary endeavour at the intersection of robotics and oceanography. The focus was not only on robotic exploration, but also on the scientific study of the plume. The test plan shed some light into the structure of the front propagation, evolving from an initial sharp variation of salinity to a situation in which the front has two sharp edges separated by a plateau, thus presenting an added difficulty to some tracking algorithms. This observation also provided insights into trajectory adaptation and, more important, to the selection of the initial location for the AUV. Space limitations preclude a thorough discussion of the results, but our parameterizable approach and the study of the conditions for parameter selection will be at the heart of a decision support system for optimized plume field studies. Future work will also use machine learning techniques to optimize tracking and mapping campaigns. Extensions to multi-AUV settings are also being considered. The proposed developments will be field tested in the next few months with the AUV described in [15].

\section{REFERENCES}

[1] J. Pinto, R. Mendes, J. C. da Silva, J. M. Dias, and J. B. de Sousa, "Multiple autonomous vehicles applied to plume detection and tracking," in 2018 OCEANS-MTS/IEEE Kobe Techno-Oceans (OTO), pp. 1-6, IEEE, 2018

[2] J. Hwang, N. Bose, and S. Fan, "Auv adaptive sampling methods: A review," Applied Sciences, vol. 9, no. 15, p. 3145, 2019.

[3] J. Jurisa, "Columbia river plume." Accessed 24-06-2021.

[4] M. Fahad, N. Saul, Y. Guo, and B. Bingham, "Robotic simulation of dynamic plume tracking by unmanned surface vessels," in 2015 IEEE International Conference on Robotics and Automation (ICRA), pp. 2654-2659, IEEE, 2015.

[5] S. Li, Y. Guo, and B. Bingham, "Multi-robot cooperative control for monitoring and tracking dynamic plumes," in 2014 IEEE International Conference on Robotics and Automation (ICRA), pp. 67-73, IEEE, sep 2014.

[6] J. Hwang, N. Bose, H. Nguyen, and G. Williams, "Auv adaptive sampling to delineate subsurface oil plumes," in 2020 IEEE/OES
Autonomous Underwater Vehicles Symposium (AUV)(50043), pp. 1-2, IEEE, 2020

[7] J. Hwang, N. Bose, H. D. Nguyen, and G. Williams, "Oil plume mapping: Adaptive tracking and adaptive sampling from an autonomous underwater vehicle," IEEE Access, vol. 8, pp. 198021-198034, 2020.

[8] Y. Zhang, R. S. McEwen, J. P. Ryan, J. G. Bellingham, H. Thomas, C. H. Thompson, and E. Rienecker, "A peak-capture algorithm used on an autonomous underwater vehicle in the 2010 gulf of mexico oil spill response scientific survey," Journal of Field Robotics, vol. 28, no. 4, pp. 484-496, 2011.

[9] J. Fonseca, J. Wei, K. H. Johansson, and T. A. Johansen, "Cooperative decentralised circumnavigation with application to algal bloom tracking," in IEEE International Conference on Intelligent Robots and Systems, pp. 3276-3281, IEEE, nov 2019.

[10] I. Belkin, J. B. D. Sousa, J. Pinto, R. Mendes, and F. Lopez-Castejon, "A new front-tracking algorithm for marine robots," in AUV 2018 - 2018 IEEE/OES Autonomous Underwater Vehicle Workshop, Proceedings, Institute of Electrical and Electronics Engineers Inc., nov 2018.

[11] Y. Zhang, M. A. Godin, J. G. Bellingham, and J. P. Ryan, "Using an autonomous underwater vehicle to track a coastal upwelling front," IEEE Journal of Oceanic Engineering, vol. 37, no. 3, pp. 338-347, 2012.

[12] C. J. Cannell, A. S. Gadre, and D. J. Stilwell, "Boundary tracking and rapid mapping of a thermal plume using an autonomous vehicle," in OCEANS 2006, pp. 1-6, IEEE, 2006.

[13] C. J. Cannell and D. J. Stilwell, "A comparison of two approaches for adaptive sampling of environmental processes using autonomous underwater vehicles," in Proceedings of OCEANS 2005 MTS/IEEE, pp. 1514-1521, IEEE, 2005.

[14] D. Kularatne, R. N. Smith, and M. A. Hsieh, "Zig-zag wanderer: Towards adaptive tracking of time-varying coherent structures in the ocean," in 2015 IEEE International Conference on Robotics and Automation (ICRA), pp. 3253-3258, IEEE, 2015.

[15] J. Pinto, P. S. Dias, R. Martins, J. Fortuna, E. Marques, and J. Sousa, "The LSTS toolchain for networked vehicle systems," in OCEANS 2013 MTS/IEEE Bergen: The Challenges of the Northern Dimension, 2013.

[16] R. Martins, P. S. Dias, E. R. Marques, J. Pinto, J. B. Sousa, and F. L. Pereira, "Imc: A communication protocol for networked vehicles and sensors," in Oceans 2009-Europe, pp. 1-6, IEEE, 2009.

[17] F. Pereira, J. Pinto, J. Sousa, R. Gomes, G. M. Gonçalves, and P. Dias, "Mission planning and specification in the neptus framework," in Proceedings 2006 IEEE International Conference on Robotics and Automation, 2006. ICRA 2006., pp. 3220-3225, IEEE, 2006.

[18] M. C. Sousa, A. S. Ribeiro, M. Des, R. Mendes, I. Alvarez, M. GomezGesteira, and J. M. Dias, "Integrated high-resolution numerical model for the nw iberian peninsula coast and main estuarine systems," Journal of Coastal Research, pp. 66-70, 2018. 